\title{
Non-commutative association schemes and their fusion association schemes
}

\author{
Hadi Kharaghani* Sho Suda ${ }^{\dagger}$
}

October 8, 2018

\begin{abstract}
We give a sufficient condition for a non-commutative association scheme to have a fusion association scheme, and construct non-commutative association schemes from symmetric balanced generalized weighing matrices and generalized Hadamard matrices. We then apply the criterion to these non-commutative association schemes to obtain symmetric fusion association schemes.
\end{abstract}

\section{Introduction}

Association schemes are considered as an abstraction of the centralizer of transitive permutation groups, and can be described as the subalgebra of the matrix algebra generated by the disjoint $(0,1)$-matrices which are closed under the transposition and their sum equals to the all-ones matrix 11, 12. Much of interest is focused on the case of multiplicity free transitive permutation groups. In such cases, the corresponding association schemes are commutative. In the present paper, we consider non-commutative association schemes obtained from some combinatorial objects such as symmetric balanced generalized weighing matrices and generalized Hadamard matrices.

Kharaghani and Torabi 9 showed that for any prime power $q$, the edge set of complete graph $K_{q^{3}+q^{2}+q+1}$ is decomposed into $q+1$ strongly regular graphs sharing $q^{2}+1$ disjoint cliques. The decomposition is based on symmetric balanced generalized weighing matrices $B G W\left(q^{2}+1, q^{2}, q^{2}-1\right)$ with zero diagonal entries over a cyclic group of order $q+1$, see 3 for details. Motivated by this decomposition, Klin, Reichard and Woldar [10] defined the concept of Siamese objects as a partition of the edge set of the complete graph, and studied it from the view point of graph theory and group theory. In particular, it was shown that an action of $P G L(2, q)$ yields a non-commutative association scheme.

In this paper it is shown that non-commutative association schemes are obtained from the following objects:

- any symmetric balanced generalized weighing matrix $B G W(n+1, n, n-1)$ with zero diagonal entries over a cyclic group $C_{m}$ of order $m$,

- a generalized Hadamard matrix attached to finite fields.

\footnotetext{
${ }^{*}$ Department of Mathematics and Computer Science, University of Lethbridge, Lethbridge, Alberta, T1K 3M4, Canada. kharaghani@uleth.ca

${ }^{\dagger}$ Department of Mathematics Education, Aichi University of Education, Kariya, Aichi 448-8542, Japan. suda@auecc.aichi-edu.ac.jp
} 
Our first example of non-commutative association scheme is obtained from the $B G W(n+$ $1, n, n-1)$ over the cyclic group $C_{m}$ with $(n, m)=\left(q^{2}, q+1\right)$, includes Kharaghani and Torabi's work, and has the parameters of the non-commutative association scheme obtained by Klin, Reichard and Woldar. We also establish a sufficient condition for a non-commutative association scheme to possess a fusion association scheme, and thus obtain an analog of a part of result by Bannai 1 and Muzychuk 11. By applying this criterion to our non-commutative association scheme we obtain some symmetric fusion association schemes. Finally, the Wedderburn decomposition (or character table) and the eigenmatrices of the association schemes are explicitly determined.

\section{Preliminaries}

Throughout this paper, $I_{n}, J_{n}$ denote the identity matrix of order $n$, the all-ones matrix of order $n$ respectively.

Let $d$ be a positive integer. Let $X$ be a finite set of size $v$ and $R_{i}(i \in\{0,1, \ldots, d\})$ be a nonempty subset of $X \times X$. The adjacency matrix $A_{i}$ of the graph with vertex set $X$ and edge set $R_{i}$ is a $v \times v(0,1)$-matrix with rows and columns indexed by $X$ such that $\left(A_{i}\right)_{x y}=1$ if $(x, y) \in R_{i}$ and $\left(A_{i}\right)_{x y}=0$ otherwise. An association scheme of $d$-class is a pair $\left(X,\left\{R_{i}\right\}_{i=0}^{d}\right)$ satisfying the following:

(i) $A_{0}=I_{v}$.

(ii) $\sum_{i=0}^{d} A_{i}=J_{v}$.

(iii) $A_{i}^{\top} \in\left\{A_{1}, \ldots, A_{d}\right\}$ for any $i \in\{1, \ldots, d\}$.

(iv) For all $i$ and $j, A_{i} A_{j}$ is a linear combination of $A_{0}, A_{1}, \ldots, A_{d}$.

We also refer to the set of non-zero $(0,1)$-matrices satisfying (i)-(iv) as an association scheme. An association scheme is symmetric if $A_{i}^{\top}=A_{i}$ holds for any $i$. An association scheme is commutative if $A_{i} A_{j}=A_{j} A_{i}$ holds for any $i, j$, non-commutative otherwise. Non-commutative association schemes are also known as homogeneous coherent configurations [5]. Note that symmetric association schemes are commutative association schemes by (iv). For a symmetric association scheme $\left(X,\left\{R_{i}\right\}_{i=0}^{2}\right)$ of class 2 , the graph with adjacency matrix $A_{i}$ for $i \in\{1,2\}$ is said to be a strongly regular graph.

Let $\left\{A_{0}, A_{1}, \ldots, A_{d}\right\}$ be a non-commutative association scheme. The vector space over $\mathbb{C}$ spanned by the $A_{i}$ 's forms a non-commutative algebra, denoted by $\mathcal{A}$ and called the adjacency algebra. Since the algebra $\mathcal{A}$ is semisimple, the adjacency algebra is isomorphic to $\oplus_{k=1}^{n} \operatorname{Mat}_{d_{k}}(\mathbb{C})$ for uniquely determined positive integers $n, d_{1}, \ldots, d_{n}$. We write $\mathcal{I}=$ $\left\{(i, j, k) \in \mathbb{N}^{3} \mid 1 \leq i, j \leq d_{k}, 1 \leq k \leq n\right\}$ where $\mathbb{N}$ denotes the set of positive integers, and call the set $\mathcal{I}$ the index set of a dual basis.

For $k \in\{1, \ldots, n\}$, let $\varphi_{k}$ be an irreducible representation from $\mathcal{A}$ to $\operatorname{Mat}_{d_{k}}(\mathbb{C})$. Despite the fact that the entries of the image of irreducible representations are not uniquely determined, the character table defined below is uniquely determined. The character table $T$ is defined to be an $n \times(d+1)$ matrix with $(k, i)$ entry equal to $\operatorname{tr}\left(\varphi_{k}\left(A_{i}\right)\right)$, where $\operatorname{tr}$ denotes the trace.

The following is due to Higman [6]. Let $E_{i, j}^{(k)}((i, j, k) \in \mathcal{I})$ be a basis of $\mathcal{A}$ such that $\varphi_{k}\left(E_{i, j}^{(k)}\right) \in \operatorname{Mat}_{d_{k}}(\mathbb{C}), E_{i, j}^{(k)} E_{i^{\prime}, j^{\prime}}^{\left(k^{\prime}\right)}=\delta_{k, k^{\prime}} \delta_{j, i^{\prime}} E_{i, j^{\prime}}^{(k)}$ and $E_{i, j}^{(k)^{*}}=E_{j, i}^{(k)}$, where $*$ denotes the transpose conjugate. Since $A_{l}(l \in\{0,1, \ldots, d\})$ and $E_{i, j}^{(k)}((i, j, k) \in \mathcal{I})$ are bases of the adjacency algebra, there exist complex numbers $p_{(i, j), l}^{(k)}$ and $q_{l,(i, j)}^{(k)}$ such that

$$
A_{l}=\sum_{(i, j, k) \in \mathcal{I}} p_{(i, j), l}^{(k)} E_{i, j}^{(k)}, \quad E_{i, j}^{(k)}=\frac{1}{v} \sum_{l=0}^{d} q_{l,(i, j)}^{(k)} A_{l} .
$$


For $k \in\{1, \ldots, n\}$, set $P_{k}=\left(p_{(i, j), l}^{(k)}\right)$ and $Q_{k}=\left(q_{l,(i, j)}^{(k)}\right)$, where $(i, j)$ runs over $\{(a, b) \mid$ $\left.1 \leq a, b \leq d_{k}\right\}$ and $l$ runs over $\{0,1, \ldots, d\}$. Note that the ordering of indices of rows of $P_{k}$ and columns of $Q_{k}$ is the lexicographical order. We then define $(d+1) \times(d+1)$ matrices $P$ and $Q$ by

$$
P=\left(\begin{array}{c}
P_{1} \\
P_{2} \\
\vdots \\
P_{n}
\end{array}\right), \quad Q=\left(\begin{array}{llll}
Q_{1} & Q_{2} & \cdots & Q_{n}
\end{array}\right)
$$

In order to derive the character table from the matrix $Q$, we prepare the following lemma.

Lemma 2.1. Let $k \in\{1, \ldots, n\}$.

(1) For any $i, j, i^{\prime}, j^{\prime} \in\left\{1, \ldots, d_{k}\right\}, \operatorname{rank} E_{i, j}^{(k)}=\operatorname{rank} E_{i^{\prime}, j^{\prime}}^{(k)}$.

(2) For any $i, j \in\left\{1, \ldots, d_{k}\right\}, \operatorname{tr} E_{i, j}^{(k)}=\delta_{i, j} \operatorname{rank} E_{i, j}^{(k)}$.

Proof. Let $i, j \in\left\{1, \ldots, d_{k}\right\}$.

(1): Since $E_{i, j}^{(k)^{*}}=E_{j, i}^{(k)}, \operatorname{rank} E_{i, j}^{(k)}=\operatorname{rank} E_{j, i}^{(k)}$. Next we use $E_{i, j}^{(k)} E_{j, i}^{(k)}=E_{i, i}^{(k)}$ to obtain $\operatorname{rank} E_{i, i}^{(k)}=\operatorname{rank} E_{i, j}^{(k)}$. This proves (1).

(2): Since $E_{i, i}^{(k)}$ is an idempotent, $\operatorname{tr} E_{i, i}^{(k)}=\operatorname{rank} E_{i, i}^{(k)}$. Next assume that $i$ is not equal to $j$. Then, taking the trace of $E_{i, j}^{(k)}=E_{i, i}^{(k)} E_{i, j}^{(k)}$, we have

$$
\operatorname{tr} E_{i, j}^{(k)}=\operatorname{tr}\left(E_{i, i}^{(k)} E_{i, j}^{(k)}\right)=\operatorname{tr}\left(E_{i, j}^{(k)} E_{i, i}^{(k)}\right)=0 .
$$

Therefore we have $\operatorname{tr} E_{i, j}^{(k)}=\delta_{i, j} \operatorname{rank} E_{i, j}^{(k)}$.

By Lemma 2.1. we let $m_{k}=\operatorname{rank} E_{i, j}^{(k)}$ for $k \in\{1, \ldots, n\}$ and any $i, j \in\left\{1, \ldots, d_{k}\right\}$. We also let $v_{l}=\frac{1}{v} \tau\left(A_{l}\right)$, where $\tau$ denotes the function assigning a matrix to the sum of the entries.

The following proposition shows how to derive the character table from the matrix $Q$. The proof is based on the same idea as in [2, Theorem 3.5 (i)].

Proposition 2.2. (1) For each $k$, the $k$-th row of $T$ is the scalar multiple by $m_{k}$ of the sum of the rows of $P_{k}$ corresponding to all indices $(i, i)$.

(2) $\Delta_{m} P=\bar{Q} \Delta_{v}$ holds, where $\bar{Q}$ is the complex conjugate of $Q, \Delta_{m}$ is the diagonal matrix indexed by $\mathcal{I}$ with $(i, j, k)$-th entry equal to $m_{k}$ and $\Delta_{v}$ is the diagonal matrix indexed by $\{0,1, \ldots, d\}$ with $i$-th entry equal to $v_{i}$.

Proof. (1): Since $\varphi_{k}\left(A_{l}\right)=\sum_{i, j=1}^{d_{k}} p_{(i, j), l}^{(k)} E_{i, j}^{(k)}$, we use Lemma 2.1 to obtain the following;

$$
\operatorname{tr}\left(\varphi_{k}\left(A_{l}\right)\right)=\sum_{i, j=1}^{d_{k}} p_{(i, j), l}^{(k)} \operatorname{tr}\left(E_{i, j}^{(k)}\right)=\sum_{i=1}^{d_{k}} p_{(i, i), l}^{(k)} m_{k}
$$

from which we obtain the desired result.

(2): Calculating $\operatorname{tr}\left(A_{l} E_{i, j}^{(k)}\right)$ in two ways as follows. On the one hand, by Lemma 2.1

$$
\begin{aligned}
\operatorname{tr}\left(A_{l} E_{i, j}^{(k)}\right) & =\operatorname{tr}\left(\sum_{i^{\prime}=1}^{d_{k}} p_{\left(i^{\prime}, i\right), l}^{(k)} E_{i^{\prime}, j}^{(k)}\right) \\
& =p_{(j, i), l}^{(k)} m_{k} .
\end{aligned}
$$


On the other hand, let $l^{\prime}$ be such that $A_{l}^{\top}=A_{l^{\prime}}$ and $\circ$ denote the entry-wise product for matrices. Since $\operatorname{tr}(A B)=\tau\left(A^{\top} \circ B\right)$ and $v_{l^{\prime}}=v_{l}$, we have

$$
\begin{aligned}
\operatorname{tr}\left(A_{l} E_{i, j}^{(k)}\right) & =\tau\left(A_{l^{\prime}} \circ E_{i, j}^{(k)}\right) \\
& =\tau\left(\frac{1}{v} q_{l^{\prime},(i, j)}^{(k)} A_{l^{\prime}}\right) \\
& =v_{l^{\prime}} q_{l^{\prime},(i, j)}^{(k)} \\
& =v_{l} q_{l,(j, i)}^{(k)},
\end{aligned}
$$

where the last equality follows from $E_{i, j}^{(k)^{*}}=E_{j, i}^{(k)}$. Thus we obtain the desired result.

\section{Bannai-Muzychuk criterion for non-commutative as- sociation schemes}

Let $\left(X,\left\{R_{i}\right\}_{i=0}^{d}\right)$ be an association scheme. Let $\left\{\Lambda_{0}, \Lambda_{1}, \ldots, \Lambda_{e}\right\}$ be a partition of $\{0,1, \ldots, d\}$ such that $\Lambda_{0}=\{0\}$. If $\left(X,\left\{\cup_{l^{\prime} \in \Lambda_{l}} R_{l^{\prime}}\right\}_{l=0}^{e}\right)$ is an association scheme, then it is called a $f u$ sion scheme of the association scheme $\left(X,\left\{R_{i}\right\}_{i=0}^{d}\right)$. The following is a result obtained by Bannai [1] and by Muzychuk 11] independently. It is a criterion characterizing the fusion scheme in terms of eigenmatrices for commutative schemes. For commutative association schemes, the index set of a dual basis $\mathcal{I}$ is of the form $\mathcal{I}=\{(1,1, k) \mid k=1, \ldots, d+1\}$. Thus we use the standard notion of the entries of the first eigenmatrix $P=\left(p_{i, j}\right)_{i, j=0}^{d+1}$ : $p_{i, j}=p_{(1,1), j}^{(i)}$ for $i, j \in\{0,1, \ldots, d\}$.

Theorem 3.1. Let $\left(X,\left\{R_{i}\right\}_{i=0}^{d}\right)$ be a commutative association scheme. Let $\left\{\Lambda_{0}, \Lambda_{1}, \ldots, \Lambda_{e}\right\}$ be a partition of $\{0,1, \ldots, d\}$ such that $\Lambda_{0}=\{0\}$. Then the following are equivalent.

(1) $\left(X,\left\{\bigcup_{l^{\prime} \in \Lambda_{l}} R_{l^{\prime}}\right\}_{l=0}^{e}\right)$ is a fusion scheme of $\left(X,\left\{R_{i}\right\}_{i=0}^{d}\right)$.

(2) (a) For any $l \in\{0,1, \ldots, e\}$ there exists $k \in\{0,1, \ldots, e\}$ such that $\sum_{l^{\prime} \in \Lambda_{l}} A_{l^{\prime}}^{\top}=$ $\sum_{k^{\prime} \in \Lambda_{k}} A_{k^{\prime}}$, and (b) there exists a partition $\left\{\Delta_{k} \mid k=0,1, \ldots, e\right\}$ of $\{0,1, \ldots, n\}$ such that $\Delta_{0}=\{0\}$ and $\sum_{l^{\prime} \in \Lambda_{l}} p_{k^{\prime}, l^{\prime}}$ does not depend on the choice of $k^{\prime} \in \Delta_{k}$.

In the following, we give a sufficient condition for a non-commutative association scheme to have a fusion scheme, an analog of one way of Theorem 3.1 in non-commutative association schemes.

Note that in the following lemma we use a specific partition of $\mathcal{I}=\left\{(i, j, k) \in \mathbb{N}^{3}\right.$ $\left.1 \leq i, j \leq d_{k}, 1 \leq k \leq n\right\}$, for the index set of a dual basis. For each $k \in\{1, \ldots, n\}$, let $\left\{I_{1}^{(k)}, \ldots, I_{f_{k}}^{(k)}\right\}$ be a partition of $\left\{1, \ldots, d_{k}\right\}$. For $a, b \in\left\{1, \ldots, f_{k}\right\}$, define $\mathcal{I}_{a, b}^{(k)}=I_{a}^{(k)} \times$ $I_{b}^{(k)}$. Then we call the partitions $\mathcal{I}_{a, b}^{(k)}\left(k \in\{1, \ldots, n\}, a, b \in\left\{1, \ldots, f_{k}\right\}\right)$ of $\left\{1, \ldots, d_{k}\right\}^{2}$ canonical.

Theorem 3.2. Let $\left(X,\left\{R_{i}\right\}_{i=0}^{d}\right)$ be an association scheme. Let $\left\{\Lambda_{0}, \Lambda_{1}, \ldots, \Lambda_{e}\right\}$ be a partition of $\{0,1, \ldots, d\}$ such that $\Lambda_{0}=\{0\}$. Assume

(a) For any $l \in\{0,1, \ldots, e\}$ there exists $k \in\{0,1, \ldots, e\}$ such that $\sum_{l^{\prime} \in \Lambda_{l}} A_{l^{\prime}}^{\top}=$ $\sum_{k^{\prime} \in \Lambda_{k}} A_{k^{\prime}}$, and

(b) there exists a canonical partition $\mathcal{I}_{a, b}^{(k)}\left(a, b \in\left\{1, \ldots, f_{k}\right\}\right)$ of $\left\{1, \ldots, d_{k}\right\}^{2}$ for any $k \in\{1, \ldots, n\}$ such that $\sum_{k=1}^{n} f_{k}^{2}=e+1$ and $\sum_{l^{\prime} \in \Lambda_{l}} p_{(i, j), l^{\prime}}^{(k)}$ does not depend on the choice of $(i, j) \in \mathcal{I}_{a, b}^{(k)}$.

Then $\left(X,\left\{\bigcup_{l^{\prime} \in \Lambda_{l}} R_{l^{\prime}}\right\}_{l=0}^{e}\right)$ is a fusion scheme of $\left(X,\left\{R_{i}\right\}_{i=0}^{d}\right)$. 
Proof. Let $\mathcal{A}^{\prime}$ be the vector space spanned by $\sum_{l^{\prime} \in \Lambda_{l}} A_{l^{\prime}}$ for $l \in\{0,1, \ldots, e\}$. Then $\mathcal{A}^{\prime}$ is closed under the transposition by (a). Consider a subalgebra $\mathcal{A}^{\prime \prime}$ generated by the matrices $\sum_{(i, j) \in \mathcal{I}_{a, b}^{(k)}} E_{i, j}^{(k)}\left(k \in\{1, \ldots, n\}, a, b \in\left\{1, \ldots, f_{k}\right\}\right)$. Letting $c_{(i, j), l}^{(k)}=\sum_{l^{\prime} \in \Lambda_{l}} p_{(i, j), l^{\prime}}^{(k)}$, we have

$$
\begin{aligned}
\sum_{k=1}^{n} \sum_{a, b=1}^{f_{k}} \sum_{(i, j) \in \mathcal{I}_{a, b}^{(k)}} c_{(i, j), l}^{(k)} E_{i, j}^{(k)} & =\sum_{k=1}^{n} \sum_{a, b=1}^{f_{k}} \sum_{(i, j) \in \mathcal{I}_{a, b}^{(k)}}\left(\sum_{l^{\prime} \in \Lambda_{l}} p_{(i, j), l^{\prime}}^{(k)}\right) E_{i, j}^{(k)} \\
& =\sum_{l^{\prime} \in \Lambda_{l}} \sum_{(i, j, k) \in \mathcal{I}} p_{(i, j), l^{\prime}}^{(k)} E_{i, j}^{(k)} \\
& =\sum_{l^{\prime} \in \Lambda_{l}} A_{l^{\prime}} .
\end{aligned}
$$

Thus the subalgebra $\mathcal{A}^{\prime \prime}$ includes the vector space $\mathcal{A}^{\prime}$. Since the dimension of $\mathcal{A}^{\prime}$ and $\mathcal{A}^{\prime \prime}$ as the vector space over $\mathbb{C}$ coincide by the assumption $\sum_{k=1}^{n} f_{k}^{2}=e+1$, we have $\mathcal{A}^{\prime}=\mathcal{A}^{\prime \prime}$. Now since $\mathcal{A}^{\prime \prime}$ is closed under the matrix multiplication, so is $\mathcal{A}^{\prime}$. Thus $\left(X,\left\{\bigcup_{l^{\prime} \in \Lambda_{l}} R_{l^{\prime}}\right\}_{l=0}^{e}\right)$ is an association scheme.

\section{Symmetric $B G W(n+1, n, n-1)$ with zero diagonal entries over the cyclic group}

In this section, we construct a non-commutative association scheme from any given symmetric $B G W(n+1, n, n-1)$ with zero diagonal entries over a cyclic group. For completion we first recall the definition and the existence of symmetric $B G W(q+1, q, q-1)$ with zero diagonal entries over a cyclic group.

Let $G$ be a multiplicatively written finite group. A balanced generalized weighing matrix with parameters $(v, k, \lambda)$ over $G$, or a $B G W(v, k, \lambda)$ over $G$, is a matrix $W=\left(w_{i j}\right)$ of order $v$ with entries from $G \cup\{0\}$ such that (i) every row of $W$ contains exactly $k$ nonzero entries and (ii) for any distinct $i, h \in\{1,2, \ldots, v\}$, every element of $G$ is contained exactly $\lambda /|G|$ times in the multiset $\left\{w_{i j} w_{h j}^{-1} \mid 1 \leq j \leq v, w_{i j} \neq 0, w_{h j} \neq 0\right\}$. A BGW with $v=k$ is said to be a generalized Hadamard matrix, which will be dealt in Section 5 . The following is a basic result of symmetric balanced generalized weighing matrices.

Lemma 4.1. 9, Lemma 3] Let $q, m, t$ be positive integers such that $q$ is a prime power, $q=m t+1, t$ is even. Then there is a symmetric $B G W(q+1, q, q-1)$ with zero diagonal entries over the cyclic group of order $m$.

Let $n, m$ be positive integers. Let $W=\left(w_{i, j}\right)_{i, j=1}^{n+1}$ be any symmetric $B G W(n+1, n, n-$ 1) with zero diagonal entries over the cyclic group $C_{m}=\langle U\rangle$ generated by the circulant matrix $U$ of order $m$ with the first row $(0,1,0, \ldots, 0)$. We construct a non-commutative association scheme from $W$ as follows.

For $l \in\{0,1, \ldots, m-1\}$, define $N_{l}$ to be an $(n+1) m \times(n+1) m(0,1)$-matrix with $m \times m$ block submatrices such that its $(i, j)$-block equals to

$$
\left[N_{l}\right]_{i j}= \begin{cases}J_{m} & \text { if } i=j, \\ w_{i j} U^{l} R & \text { if } i \neq j,\end{cases}
$$

where $\left[N_{l}\right]_{i j}$ denotes the $(i, j)$-block of $N_{l}$ and $R$ is the back diagonal matrix of order $m$. Note that $U R=R U^{-1}$, and $w_{i j} U=U w_{i j}$ since $w_{i j}$ is a power of $U$. Then the following holds.

Theorem 4.2. (1) The matrices $N_{0}, \ldots, N_{m-1}$ are symmetric matrices and share the diagonal blocks $I_{n+1} \otimes J_{m}$. 
(2) For $l \in\{0,1, \ldots, m-1\}, N_{l}\left(I_{n+1} \otimes J_{m}\right)=\left(I_{n+1} \otimes J_{m}\right) N_{l}=m I_{n+1} \otimes J_{m}+\left(J_{n+1}-\right.$ $\left.I_{n+1}\right) \otimes J_{m}$.

(3) For $l, l^{\prime} \in\{0,1, \ldots, m-1\}$,

$$
N_{l} N_{l^{\prime}}=I_{n+1} \otimes\left(m J_{m}+n U^{l-l^{\prime}}\right)+\left(2+\frac{n-1}{m}\right)\left(J_{n+1}-I_{n+1}\right) \otimes J_{m} .
$$

Proof. (1): Letting $i, j$ be distinct, the transpose of $(i, j)$-block of $N_{l}$ is

$$
\left(w_{i j} U^{l} R\right)^{\top}=R U^{-l} w_{i j}^{-1}=U^{l} R w_{i j}^{-1}=U^{l} w_{i j} R=w_{i j} U^{l} R,
$$

which is equal to the $(j, i)$-block of $N_{l}$. Thus each $N_{l}$ is symmetric. Since the off-diagonal blocks of $N_{l}$ and $N_{l^{\prime}}$ for distinct $l, l^{\prime}$ are disjoint, $N_{l}$ and $N_{l^{\prime}}$ share the diagonal blocks $I_{n+1} \otimes J_{m}$.

(2): It follows from the fact that $\left(w_{i j} U^{l} R\right) J_{m}=J_{m}\left(w_{i j} U^{l} R\right)=J_{m}$.

(3): Let $l, l^{\prime} \in\{0,1, \ldots, m-1\}$. For $i, j \in\{1, \ldots, n+1\}$ we calculate the $(i, j)$-block of $N_{l} N_{l^{\prime}}$ as follows:

$$
\begin{aligned}
{\left[N_{l} N_{l^{\prime}}\right]_{i j} } & =\sum_{k=1}^{n+1}\left(\delta_{i, k} J_{m}+w_{i k} U^{l} R\right)\left(\delta_{j, k} J_{m}+w_{j k} U^{l^{\prime}} R\right)^{\top} \\
& =\sum_{k=1}^{n+1} \delta_{i, k} \delta_{j, k} m J_{m}+\sum_{k=1}^{n+1} \delta_{i, k} w_{j k}^{\top} J_{m}+\sum_{k=1}^{n+1} \delta_{j, k} w_{i k} J_{m}+\sum_{k=1}^{n+1} w_{i k} w_{j k}^{\top} U^{l-l^{\prime}} \\
& =\delta_{i, j} m J_{m}+w_{j i}^{\top} J_{m}+w_{i j} J_{m}+\left(\delta_{i, j} n I_{m}+\left(1-\delta_{i, j}\right) \frac{n-1}{m} J_{m}\right) U^{l-l^{\prime}} \\
& =\delta_{i, j} m J_{m}+2\left(1-\delta_{i, j}\right) J_{m}+\delta_{i, j} n U^{l-l^{\prime}}+\left(1-\delta_{i, j}\right) \frac{n-1}{m} J_{m} \\
& = \begin{cases}m J_{m}+n U^{l-l^{\prime}} & \text { if } i=j, \\
\left(2+\frac{n-1}{m}\right) J_{m} & \text { if } i \neq j,\end{cases}
\end{aligned}
$$

where we used the fact that $w_{i j}$ and $U$ commute in second equality.

Remark 4.3. (1) A group divisible design with parameters $\left(v, k, m, n, \lambda_{1}, \lambda_{2}\right)$ is a pair $(V, \mathcal{B})$ where $V$ is a point set of $v$ elements and $\mathcal{B}$ is a block set of $k$-element subsets of $V$ such that the point set $V$ being decomposed into $m$ classes of size $n$ such that two distinct points from the same class are contained in exactly $\lambda_{1}$ blocks, and two points from different classes are contained in exactly $\lambda_{2}$ blocks. A group divisible design is symmetric if its dual is also a group divisible design. A $(0,1)$-matrix $A$ is the incidence matrix of a symmetric group divisible design if and only if the $(0,1)$-matrix $A$ satisfies

$$
A A^{\top}=A^{\top} A=k I_{v}+\lambda_{1}\left(I_{m} \otimes J_{n}-I_{v}\right)+\lambda_{2}\left(J_{v}-I_{m} \otimes J_{n}\right) .
$$

Theorem 4.2 shows that each $(0,1)$-matrix $N_{l}$ is a symmetric group divisible design with parameters $\left((n+1) m, n+m, n+1, m, m, 2+\frac{n-1}{m}\right)$.

(2) If $\lambda:=\lambda_{1}=\lambda_{2}$, then the group divisible design is a symmetric $2-(v, k, \lambda)$ design. When $m=2+\frac{n-1}{m}$, that is $n=(m-1)^{2}$, the symmetric group divisible designs $N_{0}, \ldots, N_{m-1}$ are symmetric $2-((n+1) m, n+m, m)$ designs sharing the diagonal blocks $I_{n+1} \otimes J_{m}$. This result is a generalization of [9, Theorem 5].

We now construct an association scheme from a symmetric $B G W(n+1, n, n-1)$ with zero diagonal entries over a cyclic group of order $m$. Define

$$
A_{l, 0}=I_{n+1} \otimes U^{l}, \quad A_{l, 1}=N_{l}-I_{n+1} \otimes J_{m}
$$

for $l=0,1, \ldots, m-1$. 
Theorem 4.4. The set of matrices $\left\{A_{l, 0}, A_{l, 1} \mid l=0,1, \ldots, m-1\right\}$ forms a noncommutative association scheme of class $2 m-1$.

Proof. The conditions (i)-(iii) in the definition of association schemes are clearly satisfied. We need to show that the condition (iv) in the definition of association schemes is satisfied. It is easy to see that

$$
A_{l, 0} A_{l^{\prime}, 0}=A_{l+l^{\prime}, 0}, \quad A_{l, 0} A_{l^{\prime}, 1}=A_{l+l^{\prime}, 1}, \quad A_{l, 1} A_{l^{\prime}, 0}=A_{l-l^{\prime}, 1},
$$

where the addition and subtraction of indices are taken in modulo $m$. Finally we calculate $A_{l, 1} A_{l^{\prime}, 1}$ for $l, l^{\prime} \in\{0,1, \ldots, m-1\}$. By Theorem 4.2,

$$
\begin{aligned}
A_{l, 1} A_{l^{\prime}, 1}= & \left(N_{l}-I_{n+1} \otimes J_{m}\right)\left(N_{l^{\prime}}-I_{n+1} \otimes J_{m}\right) \\
= & N_{l} N_{l^{\prime}}-N_{l}\left(I_{n+1} \otimes J_{m}\right)-\left(I_{n+1} \otimes J_{m}\right) N_{l^{\prime}}+m I_{n+1} \otimes J_{m} \\
= & \left(I_{n+1} \otimes\left(m J_{m}+n U^{l-l^{\prime}}\right)+\left(2+\frac{n-1}{m}\right)\left(J_{n+1}-I_{n+1}\right) \otimes J_{m}\right) \\
& \quad-2\left(m I_{n+1} \otimes J_{m}+\left(J_{n+1}-I_{n+1}\right) \otimes J_{m}\right)+m I_{n+1} \otimes J_{m} \\
= & n I_{n+1} \otimes U^{l-l^{\prime}}+\frac{n-1}{m}\left(J_{n+1}-I_{n+1}\right) \otimes J_{m} \\
= & n A_{l-l^{\prime}, 0}+\frac{n-1}{m}\left(A_{0,1}+\cdots+A_{m-1,1}\right) .
\end{aligned}
$$

Thus the condition (iv) is satisfied.

We view the cyclic group $C_{m}$ of order $m$ as the additive group $\mathbb{Z}_{m}$. Let $w=e^{2 \pi \sqrt{-1} / m}$. For $\alpha, \beta \in \mathbb{Z}_{m}$, the irreducible character denoted $\chi_{\beta}$ is $\chi_{\beta}(\alpha)=w^{\alpha \beta}$. The character table $K$ of the abelian group $\mathbb{Z}_{m}$ is an $m \times m$ matrix with rows and columns indexed by the elements of $\mathbb{Z}_{m}$ with $(\alpha, \beta)$-entry equal to $\chi_{\beta}(\alpha)$. Note that $\chi_{\beta}(\alpha)=\chi_{\alpha}(\beta)$. Then the Schur orthogonality relation shows that $K K^{\top}=m I_{m}$.

Define $F_{\alpha, 0}, F_{\alpha, 1}$ as

$$
F_{\alpha, 0}=\sum_{\gamma \in \mathbb{Z}_{m}} \chi_{\alpha}(\gamma) A_{\gamma, 0}, \quad F_{\alpha, 1}=\sum_{\gamma \in \mathbb{Z}_{m}} \chi_{\alpha}(\gamma) A_{\gamma, 1}
$$

Using the intersection numbers described in Theorem 4.4 the following lemma is easy to see.

Lemma 4.5. The matrices $F_{\alpha, 0}, F_{\alpha, 1}\left(\alpha \in \mathbb{Z}_{m}\right)$ satisfy the following equations; for $\alpha, \beta \in$ $\mathbb{Z}_{m}$,

$$
\begin{aligned}
& F_{\alpha, 0} F_{\beta, 0}=\delta_{\alpha, \beta} m F_{\alpha, 0}, \\
& F_{\alpha, 1} F_{\beta, 1}=\delta_{\alpha,-\beta} n m F_{\alpha, 0}+\delta_{\alpha, 0} \delta_{\beta, 0} m(n-1) F_{0,1}, \\
& F_{\alpha, 0} F_{\beta, 1}=\delta_{\alpha, \beta} m F_{\alpha, 1}, \\
& F_{\alpha, 1} F_{\beta, 0}=\delta_{\alpha,-\beta} m F_{\alpha, 1} .
\end{aligned}
$$


For $i \in\{0,1,2,3\}, j, k \in\{1,2\}, \alpha \in \mathbb{Z}_{m}$, let $E_{i}, E_{j, k}^{(\alpha)}$ be

$$
\begin{aligned}
E_{0} & =\frac{1}{(n+1) m} J_{(n+1) m}, \\
E_{1} & =\frac{1}{(n+1) m}\left(n F_{0,0}-F_{0,1}\right), \\
E_{2} & =\frac{1}{2 m}\left(F_{m / 2,0}+\frac{1}{\sqrt{n}} F_{m / 2,1}\right), \\
E_{3} & =\frac{1}{2 m}\left(F_{m / 2,0}-\frac{1}{\sqrt{n}} F_{m / 2,1}\right), \\
E_{1,1}^{(\alpha)} & =\frac{1}{m} F_{\alpha, 0}, \quad E_{2,2}^{(\alpha)}=\frac{1}{m} F_{-\alpha, 0}, \quad E_{1,2}^{(\alpha)}=\frac{1}{m} F_{\alpha, 1}, \quad E_{2,1}^{(\alpha)}=\frac{1}{m} F_{-\alpha, 1},
\end{aligned}
$$

where $E_{2}, E_{3}$ are defined only for the case $m$ even.

Theorem 4.6. (1) If $m$ is even, then the matrices $E_{0}, E_{1}, E_{2}, E_{3}, E_{1,1}^{(\alpha)}, E_{1,2}^{(\alpha)}, E_{2,1}^{(\alpha)}, E_{2,2}^{(\alpha)}$, $\alpha \in\{1, \ldots, m / 2-1\}$, provide the Wedderburn decomposition of the adjacency algebra of the association scheme.

(2) If $m$ is odd, then the matrices $E_{0}, E_{1}, E_{1,1}^{(\alpha)}, E_{1,2}^{(\alpha)}, E_{2,1}^{(\alpha)}, E_{2,2}^{(\alpha)}, \alpha \in\{1, \ldots,(m-1) / 2\}$, provide the Wedderburn decomposition of the adjacency algebra of the association scheme.

Proof. Both cases readily follow from Lemma 4.5

Remark 4.7. (1) If $m$ is even, then the adjacency algebra is isomorphic to $\oplus_{k=1}^{4+(m-2) / 2} \operatorname{Mat}_{d_{k}}(\mathbb{C})$ where $\left(d_{k}\right)_{k=1}^{4+(m-2) / 2}=(1,1,1,1,2, \ldots, 2)$ with

$$
\begin{aligned}
Q_{1} & =\left(\begin{array}{c}
\chi_{0} \\
\chi_{0}
\end{array}\right), Q_{2}=\left(\begin{array}{c}
n \chi_{0} \\
-\chi_{0}
\end{array}\right), \\
Q_{3} & =\frac{n+1}{2}\left(\begin{array}{c}
\chi_{m / 2} \\
\frac{1}{\sqrt{n}} \chi_{m / 2}
\end{array}\right), Q_{4}=\frac{n+1}{2}\left(\begin{array}{c}
\chi_{m / 2} \\
-\frac{1}{\sqrt{n}} \chi_{m / 2}
\end{array}\right), \\
Q_{k+4} & =(n+1)\left(\begin{array}{cccc}
\chi_{k} & \mathbf{0} & \chi_{m-k} & \mathbf{0} \\
\mathbf{0} & \chi_{k} & \mathbf{0} & \chi_{m-k}
\end{array}\right),
\end{aligned}
$$

for $k=1, \ldots, m / 2-1$, where $\mathbf{0}$ denotes the column zero vector.

(2) If $m$ is odd, then the adjacency algebra is isomorphic to $\oplus_{k=1}^{2+(m-1) / 2} \operatorname{Mat}_{d_{k}}(\mathbb{C})$ where $\left(d_{k}\right)_{k=1}^{2+(m-1) / 2}=(1,1,2, \ldots, 2)$ with

$$
\begin{aligned}
Q_{1} & =\left(\begin{array}{l}
\chi_{0} \\
\chi_{0}
\end{array}\right), Q_{2}=\left(\begin{array}{c}
n \chi_{0} \\
-\chi_{0}
\end{array}\right), \\
Q_{k+2} & =(n+1)\left(\begin{array}{cccc}
\chi_{k} & \mathbf{0} & \chi_{m-k} & \mathbf{0} \\
\mathbf{0} & \chi_{k} & \mathbf{0} & \chi_{m-k}
\end{array}\right),
\end{aligned}
$$

for $k=1, \ldots,(m-1) / 2$.

As corollaries of Theorem 4.4 we have the following.

Corollary 4.8. Let $T$ be the character table of the non-commutative association scheme in Theorem 4.4. 
(1) If $m$ is even, then

$$
T=\left(\begin{array}{cc}
\chi_{0}^{\top} & n \chi_{0}^{\top} \\
\chi_{0}^{\top} & -\chi_{0}^{\top} \\
\chi_{m}^{\top} / 2 & \sqrt{n} \chi_{m / 2}^{\top} \\
\chi_{m / 2}^{\top} & -\sqrt{n} \chi_{m / 2}^{\top} \\
\chi_{1}^{\top}+\chi_{m-1}^{\top} & \mathbf{0}^{\top} \\
\chi_{2}^{\top}+\chi_{m-2}^{\top} & \mathbf{0}^{\top} \\
\vdots & \vdots \\
\chi_{m / 2-1}^{\top}+\chi_{m / 2+1}^{\top} & \mathbf{0}^{\top}
\end{array}\right) .
$$

(2) If $m$ is odd, then

$$
T=\left(\begin{array}{cc}
\chi_{0}^{\top} & n \chi_{0}^{\top} \\
\chi_{0}^{\top} & -\chi_{0}^{\top} \\
\chi_{1}^{\top}+\chi_{m-1}^{\top} & \mathbf{0}^{\top} \\
\chi_{2}^{\top}+\chi_{m-2}^{\top} & \mathbf{0}^{\top} \\
\vdots & \vdots \\
\chi_{(m-1) / 2}^{\top}+\chi_{(m+1) / 2}^{\top} & \mathbf{0}^{\top}
\end{array}\right)
$$

Proof. This follows from Proposition 2.2 and Theorem 4.4 .

Corollary 4.9. (1) If $m$ is even, then the set of matrices $\left\{A_{0,0}, A_{i, 0}+A_{m-i, 0}, A_{m / 2,0}, A_{0,1}, A_{i, 1}+\right.$ $\left.A_{m-i, 1}, A_{m / 2,1} \mid i=1, \ldots, m / 2-1\right\}$ is a symmetric association scheme with the second eigenmatrix $Q$

$$
Q=\left(\begin{array}{ccccccc}
\chi_{0} & n \chi_{0} & \frac{n+1}{2} \chi_{m / 2} & \frac{n+1}{2} \chi_{m / 2} & \ldots & \frac{n+1}{2}\left(\chi_{k}+\chi_{m-k}\right) & \cdots \\
\chi_{0} & -\chi_{0} & \frac{n+1}{2 \sqrt{n}} \chi_{m / 2} & -\frac{n+1}{2 \sqrt{n}} \chi_{m / 2} & \cdots & \frac{n+1}{2}\left(\chi_{k}+\chi_{m-k}\right) & \ldots
\end{array}\right)
$$

where $k$ runs over $\{1, \ldots, m / 2-1\}$.

(2) If $m$ is odd, then the set of matrices $\left\{A_{0,0}, A_{i, 0}+A_{m-i, 0}, A_{0,1}, A_{i, 1}+A_{m-i, 1} \mid i=\right.$ $1, \ldots,(m-1) / 2\}$ is a symmetric association scheme with the second eigenmatrix $Q$

$$
Q=\left(\begin{array}{ccccc}
\chi_{0} & n \chi_{0} & \cdots & \frac{n+1}{2}\left(\chi_{k}+\chi_{m-k}\right) & \cdots \\
\chi_{0} & -\chi_{0} & \cdots & \frac{n+1}{2}\left(\chi_{k}+\chi_{m-k}\right) & \cdots
\end{array}\right)
$$

where $k$ runs over $\{1, \ldots,(m-1) / 2\}$.

Proof. The results follow from Theorem 3.2

\section{$5 \quad$ Generalized Hadamard matrices}

In 8, Kharaghani, Sasani and Suda considered symmetric association schemes attached to the finite fields of characteristic two. In this section, we consider non-commutative association schemes attached to the finite fields of odd characteristic.

Let $q=p^{m}$ be an odd prime power with $p$ an odd prime. We denote by $\mathbb{F}_{q}$ the finite field of $q$ elements. Let $H_{q}$ be the multiplicative table of $\mathbb{F}_{q}$, i.e., $H_{q}$ is a $q \times q$ matrix with rows and columns indexed by the elements of $\mathbb{F}_{q}$ with $(\alpha, \beta)$-entry equal to $\alpha \cdot \beta$. Then the matrix $H_{q}$ is a generalized Hadamard matrix with parameters $(q, 1)$ over the additive group of $\mathbb{F}_{q}$.

Let $\phi$ be a permutation representation of the additive group of $\mathbb{F}_{q}$ defined as follows. Since $q=p^{m}$, we view the additive group of $\mathbb{F}_{q}$ as $\mathbb{F}_{p}^{m}$. Again let $U$ be the circulant matrix 
of order $p$ with the first row $(0,1,0, \ldots, 0)$, and a group homomorphism $\phi: \mathbb{Z}_{p}^{m} \rightarrow G L_{q}(\mathbb{R})$ as $\phi\left(\left(x_{i}\right)_{i=1}^{m}\right)=\otimes_{i=1}^{m} U^{x_{i}}$.

From the generalized Hadamard matrix $H_{q}$ and the permutation representation $\phi$, we construct $q^{2}$ auxiliary matrices; for each $\alpha, \alpha^{\prime} \in \mathbb{F}_{q}$, define a $q^{2} \times q^{2}(0,1)$-matrix $C_{\alpha, \alpha^{\prime}}$ to be

$$
C_{\alpha, \alpha^{\prime}}=\left(\phi\left(\alpha\left(-\beta+\beta^{\prime}\right)+\alpha^{\prime}\right)\right)_{\beta, \beta^{\prime} \in \mathbb{F}_{q}}
$$

Letting $x$ be an indeterminate, we define $C_{x, \alpha}$ by $C_{x, \alpha}=J_{q^{2}}-I_{q} \otimes J_{q}$ for $\alpha \in \mathbb{F}_{q}$.

It is known that a symmetric Latin square of order $v$ with constant diagonal exists for any positive even integer $v$, see [7]. Let $L=\left(L\left(a, a^{\prime}\right)\right)_{a, a^{\prime} \in S}$ be a symmetric Latin square of order $q+1$ on the symbol set $S=\mathbb{F}_{q} \cup\{x\}$ with constant diagonal $x$. Write $L$ as $L=\sum_{a \in S} a \cdot P_{a}$, where $P_{a}$ is a symmetric permutation matrix of order $q+1$. Note that $P_{x}=I_{q+1}$.

From the $(0,1)$-matrices $C_{\alpha, \alpha^{\prime}}$ 's and the Latin square $L$, we construct divisible design graphs 4, that is a symmetric group divisible designs which is adjacency matrices of a graph, as follows. Let $R$ be the back identity matrix of order $q^{2}$. For $\alpha \in \mathbb{F}_{q}$, we define a $(q+1) q^{2} \times(q+1) q^{2}(0,1)$-matrix $N_{\alpha}$ to be

$N_{\alpha}=\left(C_{L\left(a, a^{\prime}\right), \alpha}\left(\delta_{a, a^{\prime}} I_{q^{2}}+\left(1-\delta_{a, a^{\prime}}\right) R\right)\right)_{a, a^{\prime} \in S}=I_{q+1} \otimes\left(J_{q^{2}}-I_{q} \otimes J_{q}\right)+\sum_{a \in \mathbb{F}_{q}} P_{a} \otimes C_{a, \alpha} R$.

In order to show that each $N_{\alpha}$ is a divisible design graph and study more properties, we prepare a lemma on $C_{\alpha, \alpha^{\prime}}$ and $P_{a}$.

Lemma 5.1. (1) For $\alpha \in \mathbb{F}_{q}, \sum_{a \in \mathbb{F}_{q}} C_{a, \alpha}=q I_{q} \otimes \phi(\alpha)+\left(J_{q}-I_{q}\right) \otimes J_{q}$.

(2) For $a \in \mathbb{F}_{q}$ and $\alpha, \alpha^{\prime} \in \mathbb{F}_{q}, C_{a, \alpha} C_{a, \alpha^{\prime}}=q C_{a, \alpha+\alpha^{\prime}}$.

(3) For distinct $a, a^{\prime} \in \mathbb{F}_{q}$ and $\alpha, \alpha^{\prime} \in \mathbb{F}_{q}, C_{a, \alpha} C_{a^{\prime}, \alpha^{\prime}}=J_{q^{2}}$.

(4) $\left(J_{q^{2}}-I_{q} \otimes J_{q}\right) C_{a, \alpha}=C_{a, \alpha}\left(J_{q^{2}}-I_{q} \otimes J_{q}\right)=(q-1) J_{q^{2}}$.

(5) $C_{a, \alpha} R=R C_{a,-\alpha}$.

(6) $\sum_{a, b \in \mathbb{F}_{q}, a \neq b} P_{a} P_{b}=(q-1)\left(J_{q+1}-I_{q+1}\right)$.

Proof. (1): For $\alpha, \beta, \beta^{\prime} \in \mathbb{F}_{q}$, the $\left(\beta, \beta^{\prime}\right)$-entry of $\sum_{\gamma \in \mathbb{F}_{q}} C_{\gamma, \alpha}$ is

$$
\begin{aligned}
\sum_{\gamma \in \mathbb{F}_{q}} \phi\left(\gamma\left(-\beta+\beta^{\prime}\right)+\alpha\right) & = \begin{cases}\sum_{\gamma \in \mathbb{F}_{q}} \phi(\alpha) & \text { if } \beta=\beta^{\prime} \\
\sum_{\gamma^{\prime} \in \mathbb{F}_{q}} \phi\left(\gamma^{\prime}+\alpha\right) & \text { if } \beta \neq \beta^{\prime}\end{cases} \\
& = \begin{cases}q \phi(\alpha) & \text { if } \beta=\beta^{\prime}, \\
J_{q} & \text { if } \beta \neq \beta^{\prime},\end{cases}
\end{aligned}
$$

which yields $\sum_{a \in \mathbb{F}_{q}} C_{a, \alpha}=q I_{q} \otimes \phi(\alpha)+\left(J_{q}-I_{q}\right) \otimes J_{q}$.

(2): For $a, \beta, \beta^{\prime} \in \mathbb{F}_{q}$, the $\left(\beta, \beta^{\prime}\right)$-entry of $C_{a, \alpha} C_{a, \alpha^{\prime}}$ is

$$
\begin{aligned}
\sum_{\gamma \in \mathbb{F}_{q}} \phi(a(-\beta+\gamma)+\alpha) \phi\left(a\left(-\gamma+\beta^{\prime}\right)+\alpha^{\prime}\right) & =\sum_{\gamma \in \mathbb{F}_{q}} \phi\left(a\left(-\beta+\beta^{\prime}\right)+\alpha+\alpha^{\prime}\right) \\
& =q \phi\left(a\left(-\beta+\beta^{\prime}\right)+\alpha+\alpha^{\prime}\right) .
\end{aligned}
$$

Thus we have $C_{a, \alpha} C_{a, \alpha^{\prime}}=q C_{a, \alpha+\alpha^{\prime}}$.

(3): It follows from a similar calculation to (ii) with the fact that $\left\{\left(a-a^{\prime}\right) \gamma \mid \gamma \in\right.$ $\left.\mathbb{F}_{q}\right\}=\mathbb{F}_{q}$. 
(4) and (5) are easy to see, and (6) follows from the equations below. Recall that $S=\mathbb{F}_{q} \cup\{x\}$.

$$
\begin{aligned}
\sum_{a, b \in \mathbb{F}_{q}, a \neq b} P_{a} P_{b} & =\sum_{a \in \mathbb{F}_{q}} P_{a}\left(\sum_{b \in S \backslash\{x, a\}} P_{b}\right) \\
& =\sum_{a \in \mathbb{F}_{q}} P_{a}\left(J_{q+1}-I_{q+1}-P_{a}\right) \\
& =\sum_{a \in \mathbb{F}_{q}}\left(J_{q+1}-P_{a}-I_{q+1}\right) \\
& =q\left(J_{q+1}-I_{q+1}\right)-\sum_{a \in \mathbb{F}_{q}} P_{a} \\
& =(q-1)\left(J_{q+1}-I_{q+1}\right) .
\end{aligned}
$$

Now we are ready to prove the results for $N_{\alpha}$ 's.

Theorem 5.2. (1) For any $\alpha \in \mathbb{F}_{q}, N_{\alpha}$ is symmetric.

(2) For any $\alpha, \beta \in \mathbb{F}_{q}$,

$$
N_{\alpha} N_{\beta}=q^{2} I_{q+1} \otimes I_{q} \otimes \phi(\alpha-\beta)+\left(q^{2}-4 q+3\right) I_{q+1} \otimes J_{q^{2}}+3(q-1) J_{(q+1) q^{2}} .
$$

In particular, $N_{\alpha}^{2}=q^{2} I_{(q+1) q^{2}}+\left(q^{2}-4 q+3\right) I_{q+1} \otimes J_{q^{2}}+3(q-1) J_{(q+1) q^{2}}$.

Proof. (1): It follows from the properties that the matrices $P_{a}$ and $C_{a, \alpha} R$ are symmetric for $a \in \mathbb{F}_{q}$ and $\alpha \in \mathbb{F}_{q}$.

(2): We use Lemma 5.1 to obtain:

$$
\begin{aligned}
N_{\alpha} N_{\beta}= & \left(I_{q+1} \otimes\left(J_{q^{2}}-I_{q} \otimes J_{q}\right)+\sum_{a \in \mathbb{F}_{q}} P_{a} \otimes C_{a, \alpha} R\right)\left(I_{q+1} \otimes\left(J_{q^{2}}-I_{q} \otimes J_{q}\right)+\sum_{b \in \mathbb{F}_{q}} P_{b} \otimes C_{b, \beta} R\right) \\
= & I_{q+1} \otimes\left(q I_{q} \otimes J_{q}+q(q-2) J_{q^{2}}\right)+\sum_{a \in \mathbb{F}_{q}} P_{a} \otimes\left(\left(J_{q^{2}}-I_{q} \otimes J_{q}\right) C_{a, \alpha} R+C_{a, \beta} R\left(J_{q^{2}}-I_{q} \otimes J_{q}\right)\right) \\
& +\sum_{a, b \in \mathbb{F}_{q}} P_{a} P_{b} \otimes C_{a, \alpha} R C_{b, \beta} R \\
= & I_{q+1} \otimes\left(q I_{q} \otimes J_{q}+q(q-2) J_{q^{2}}\right)+\sum_{a \in \mathbb{F}_{q}} P_{a} \otimes 2(q-1) J_{q^{2}}+\sum_{a, b \in \mathbb{F}_{q}} P_{a} P_{b} \otimes C_{a, \alpha} C_{b,-\beta} R^{2} \\
= & I_{q+1} \otimes\left(q I_{q} \otimes J_{q}+q(q-2) J_{q^{2}}\right)+2(q-1)\left(J_{q+1}-I_{q+1}\right) \otimes J_{q^{2}}+\sum_{a, b \in \mathbb{F}_{q}} P_{a} P_{b} \otimes C_{a, \alpha} C_{b,-\beta} \\
= & q I_{q+1} \otimes I_{q} \otimes J_{q}+\left(q^{2}-4 q+2\right) I_{q+1} \otimes J_{q^{2}}+2(q-1) J_{q+1} \otimes J_{q^{2}}+\sum_{a, b \in \mathbb{F}_{q}} P_{a} P_{b} \otimes C_{a, \alpha} C_{b,-\beta} .
\end{aligned}
$$

The third term of the above is

$$
\begin{aligned}
& \sum_{a \in \mathbb{F}_{q}} P_{a}^{2} \otimes C_{a, \alpha} C_{a,-\beta}+\sum_{a, b \in \mathbb{F}_{q}, a \neq b} P_{a} P_{b} \otimes C_{a, \alpha} C_{b,-\beta} \\
& \sum_{a \in \mathbb{F}_{q}} I_{q+1} \otimes q C_{a, \alpha-\beta}+\sum_{a, b \in \mathbb{F}_{q}, a \neq b} P_{a} P_{b} \otimes J_{q^{2}} \\
& =q I_{q+1} \otimes\left(q I_{q} \otimes \phi(\alpha+\beta)+\left(J_{q}-I_{q}\right) \otimes J_{q}\right)+(q-1)\left(J_{q+1}-I_{q+1}\right) \otimes J_{q^{2}} \\
& =q^{2} I_{q+1} \otimes I_{q} \otimes \phi(\alpha-\beta)+I_{q+1} \otimes J_{q^{2}}-q I_{q+1} \otimes I_{q} \otimes J_{q}+(q-1) J_{(q+1) q^{2}} .
\end{aligned}
$$

Therefore

$$
N_{\alpha} N_{\beta}=q^{2} I_{q+1} \otimes I_{q} \otimes \phi(\alpha-\beta)+\left(q^{2}-4 q+3\right) I_{q+1} \otimes J_{q^{2}}+3(q-1) J_{(q+1) q^{2}} .
$$


We define $(0,1)$-matrices $A_{\alpha, i}\left(\alpha \in \mathbb{F}_{q}, i \in\{0,1\}\right)$ and $A_{2}$ as

$$
\begin{aligned}
A_{\alpha, 0} & =I_{q(q+1)} \otimes \phi(\alpha), \\
A_{\alpha, 1} & =N_{\alpha}-I_{q+1} \otimes\left(J_{q^{2}}-I_{q} \otimes J_{q}\right), \\
A_{2} & =I_{q+1} \otimes\left(J_{q^{2}}-I_{q} \otimes J_{q}\right) .
\end{aligned}
$$

Note that $A_{0,0}=I_{(q+2) q^{2}}$.

Theorem 5.3. The set of matrices $\left\{A_{\alpha, 0}, A_{\alpha, 1}, A_{2} \mid \alpha \in \mathbb{F}_{q}\right\}$ forms a non-commutative association scheme.

Proof. By the definition of $N_{\alpha}, A_{\alpha, 1}$ 's are non-zero $(0,1)$-matrices such that $\sum_{\alpha \in \mathbb{F}_{q}}\left(A_{\alpha, 0}+\right.$ $\left.A_{\alpha, 1}\right)+A_{2}=J_{(q+1) q^{2}}$. Each of $A_{\alpha, 1}$ and $A_{2}$ is symmetric, and $A_{\alpha, 0}^{\top}=A_{-\alpha, 0}$. We are now going to show that $\mathcal{A}:=\operatorname{span}_{\mathbb{C}}\left\{A_{\alpha, i}, A_{2} \mid \alpha \in \mathbb{F}_{q}, i \in\{0,1\}\right\}$ is closed under the matrix multiplication. For $\alpha, \beta \in \mathbb{F}_{q}$, the following are easy to see:

$$
\begin{aligned}
A_{\alpha, 0} A_{\beta, 0} & =A_{\alpha+\beta, 0}, \\
A_{\alpha, 0} A_{\beta, 1} & =A_{\beta, 1} A_{\alpha, 0}=A_{\alpha+\beta, 1}, \\
A_{\alpha, 0} A_{2} & =A_{2} A_{\alpha, 0}=A_{2}, \\
A_{2}^{2} & =\left(q^{2}-2 q\right) I_{q+1} \otimes J_{q^{2}}+q I_{q+1} \otimes I_{q} \otimes J_{q} .
\end{aligned}
$$

By Lemma 5.1(iv),

$$
A_{\alpha, 1} A_{2}=A_{2} A_{\alpha, 1}=\sum_{\alpha \in \mathbb{F}_{q}} A_{\alpha, 1} .
$$

Finally by Theorem 5.2 (ii),

$$
\begin{aligned}
A_{\alpha, 1} A_{\beta, 1}= & \left(N_{\alpha}-A_{2}\right)\left(N_{\beta}-A_{2}\right) \\
= & N_{\alpha} N_{\beta}-N_{\alpha} A-A_{2} N_{\beta}+A_{2}^{2} \\
= & q^{2} I_{q+1} \otimes I_{q} \otimes \phi(\alpha-\beta)+\left(q^{2}-4 q+3\right) I_{q+1} \otimes J_{q^{2}}+3(q-1) J_{(q+1) q^{2}} \\
& -2(q-1) \sum_{\alpha \in \mathbb{F}_{q}} A_{\alpha, 1}-A_{2}^{2} \\
= & q^{2} I_{q+1} \otimes I_{q} \otimes \phi(\alpha-\beta)+q I_{q+1} \otimes\left(J_{q}-I_{q}\right) \otimes J_{q}+(q-1)\left(J_{q+1}-I_{q+1}\right) \otimes J_{q^{2}} .
\end{aligned}
$$

Therefore $\mathcal{A}$ is closed under the matrix multiplication.

We view $\mathbb{F}_{q}=\mathbb{Z}_{p}^{m}$ as the additive group. For $\alpha=\left(\alpha_{1}, \ldots, \alpha_{m}\right), \beta=\left(\beta_{1}, \ldots, \beta_{m}\right) \in \mathbb{Z}_{p}^{m}$, the inner product is defined by $\langle\alpha, \beta\rangle=\alpha_{1} \beta_{1}+\cdots+\alpha_{m} \beta_{m}$. For $\beta \in \mathbb{Z}_{p}^{m}$, the irreducible character denoted $\chi_{\beta}$ is $\chi_{\beta}(\alpha)=w^{\langle\alpha, \beta\rangle}$ where $\alpha \in \mathbb{Z}_{p}^{m}, w=e^{2 \pi \sqrt{-1} / p}$. The character table $K$ of the abelian group $\mathbb{Z}_{p}^{m}$ is a $p^{m} \times p^{m}$ matrix with rows and columns indexed by the elements of $\mathbb{Z}_{p}^{m}$ with $(\alpha, \beta)$-entry equal to $\chi_{\beta}(\alpha)$. Note that $\chi_{\beta}(\alpha)=\chi_{\alpha}(\beta)$. Then the Schur orthogonality relation shows that $K K^{\top}=p^{m} I_{p^{m}}$.

To describe the primitive idempotents, let $F_{\alpha, i}, \alpha \in \mathbb{F}_{q}, i \in\{0,1\}$, be

$$
F_{\alpha, i}=\sum_{\gamma \in \mathbb{F}_{q}} \chi_{\alpha}(\gamma) A_{\gamma, i}
$$

Lemma 5.4. Let $\alpha, \beta \in \mathbb{F}_{q}$. The following hold.

(1) $F_{\alpha, 0} F_{\beta, 0}=q \delta_{\alpha, \beta} F_{\alpha, 0}$.

(2) $F_{\alpha, 0} F_{\beta, 1}=F_{\alpha, 1} F_{\beta, 0}=q \delta_{\alpha, \beta} F_{\alpha, 1}$. 
(3) $F_{\alpha, 1} F_{\beta, 1}=q^{3} \delta_{\alpha, \beta} F_{\alpha, 0}+\delta_{\alpha, 0} \delta_{\beta, 0}\left((q-1) q^{2} F_{0,1}+q^{3} A_{2}\right)$.

(4) $F_{0,0} A_{2}=A_{2} F_{0,0}=q I_{q+1} \otimes\left(J_{q^{2}}-I_{q} \otimes J_{q}\right)$.

(5) $F_{0,1} A_{2}=A_{2} F_{0,1}=\left(q^{2}-q\right) F_{0,1}$.

(6) If $\alpha \in \mathbb{F}_{q}^{*}, F_{\alpha, 0} A_{2}=A_{2} F_{\alpha, 0}=F_{\alpha, 1} A_{2}=A_{2} F_{\alpha, 1}=O$.

For $i \in\{0,1,2\}, j, k \in\{1,2\}, \alpha \in \mathbb{F}_{q}^{*}$, let $E_{i}, E_{j, k}^{(\alpha)}$ be

$$
\begin{aligned}
E_{0} & =\frac{1}{(q+1) q^{2}} J_{(q+1) q^{2}}, \\
E_{1} & =\frac{1}{(q+1) q^{2}}\left(\left(q^{2}-1\right) F_{0,0}-(q+1) A_{2}\right), \\
E_{2} & =\frac{1}{(q+1) q^{2}}\left(q F_{0,0}-F_{0,1}+q A_{2}\right), \\
E_{1,1}^{(\alpha)} & =\frac{1}{q} F_{\alpha, 0}, \quad E_{2,2}^{(\alpha)}=\frac{1}{q} F_{-\alpha, 0}, \quad E_{1,2}^{(\alpha)}=\frac{1}{q^{2}} F_{\alpha, 1}, \quad E_{2,1}^{(\alpha)}=\frac{1}{q^{2}} F_{-\alpha, 1} .
\end{aligned}
$$

Theorem 5.5. Let $S$ be any subset of $\mathbb{F}_{q}^{*}$ such that $S \cup(-S)=\mathbb{F}_{q}^{*}$ and $S \cap(-S)=\emptyset$. The matrices $E_{0}, E_{1}, E_{2}, E_{1,1}^{(\alpha)}, E_{1,2}^{(\alpha)}, E_{2,1}^{(\alpha)}, E_{2,2}^{(\alpha)}, \alpha \in S$, provide the Wedderburn decomposition of the adjacency algebra of the association scheme.

Proof. By Lemma 5.4(i)-(iii), the matrices $E_{i, j}^{(\alpha)}$ satisfy $E_{i, j}^{(\alpha)} E_{j, k}^{(\beta)}=\delta_{\alpha, \beta} \delta_{j, k} E_{i, k}^{(\alpha)}$. Also from Lemma 5.4(iv) and (v), it follows that $E_{0}, E_{1}, E_{2}$ are mutually orthogonal idempotents. Finally the orthogonality between $E_{i}$ and $E_{j, k}^{(\alpha)}$ follows from Lemma 5.4(vi).

Remark 5.6. The adjacency algebra of the association scheme in Theorem 5.3 is isomorphic to $\oplus_{k=1}^{3+(q-1) / 2} \operatorname{Mat}_{d_{k}}(\mathbb{C})$ where $\left(d_{k}\right)_{k=1}^{3+(q-1) / 2}=(1,1,1,2, \ldots, 2)$ with

$$
\begin{aligned}
Q_{1} & =\left(\begin{array}{c}
\chi_{0} \\
\chi_{0} \\
1
\end{array}\right), Q_{2}=\left(\begin{array}{c}
\left(q^{2}-1\right) \chi_{0} \\
\mathbf{0} \\
-(q+1)
\end{array}\right), Q_{3}=\left(\begin{array}{c}
q \chi_{0} \\
-\chi_{0} \\
q
\end{array}\right), \\
Q_{\alpha} & =(q+1)\left(\begin{array}{cccc}
q \chi_{\alpha} & \mathbf{0} & q \chi_{-\alpha} & \mathbf{0} \\
\mathbf{0} & \chi_{\alpha} & \mathbf{0} & \chi_{-\alpha} \\
0 & 0 & 0 & 0
\end{array}\right),
\end{aligned}
$$

for $\alpha \in S$, where $\mathbf{0}$ denotes the column zero vector.

As corollaries of Theorem 5.3 we have the following.

Corollary 5.7. Let $T$ be the character table of the non-commutative association scheme in Theorem 5.3. Then

$$
T=\left(\begin{array}{ccc}
\chi_{0}^{\top} & q^{2} \chi_{0}^{\top} & q^{2}-q \\
\chi_{0}^{\top} & -q^{2} \chi_{0}^{\top} & -\frac{q^{2}-q}{q+1} \\
\chi_{0}^{\top} & -q \chi_{0}^{\top} & q^{2}-q \\
\chi_{\alpha}^{\top}+\chi_{-\alpha}^{\top} & \mathbf{0}^{\top} & 0
\end{array}\right) .
$$

Proof. Follows from Proposition 2.2 and Theorem 4.4.

Corollary 5.8. Let $S$ be any subset of $\mathbb{F}_{q}^{*}$ such that $S \cup(-S)=\mathbb{F}_{q}^{*}$ and $S \cap(-S)=\emptyset$. The set of matrices $\left\{A_{0,0}, A_{\alpha, 0}+A_{-\alpha, 0}, A_{0,1}, A_{\alpha, 1}+A_{-\alpha, 1}, A_{2} \mid \alpha \in S\right\}$ forms a symmetric association scheme with the second eigenmatrix $Q$

$$
Q=\left(\begin{array}{cccc}
\chi_{0} & \left(q^{2}-1\right) \chi_{0} & q \chi_{0} & \frac{q(q+1)}{2}\left(\chi_{\alpha}+\chi_{-\alpha}\right) \\
\chi_{0} & \mathbf{0} & -\chi_{0} & \frac{q+1}{2}\left(\chi_{\alpha}+\chi_{-\alpha}\right) \\
1 & -(q+1) & q & 0
\end{array}\right)
$$

where $\alpha$ runs over the set $S$. 
Proof. The result follows from Theorem 3.2 .

\section{Acknowledgement}

Hadi Kharaghani is supported by an NSERC Discovery Grant. Sho Suda is supported by JSPS KAKENHI Grant Number 15K21075. Th authors thank Keiji Ito for informing us errors in the previous version and the anonymous referee for valuable comments.

\section{References}

[1] E. Bannai, Subschemes of some association schemes, J. Algebra 144 (1991), 167-188.

[2] E. Bannai, T. Ito, Algebraic Combinatorics I: Association Schemes, Benjamin/Cummings, Menro Park, CA, 1984.

[3] P.B. Gibbons, R. Mathon, Construction methods for Bhaskar Rao and related designs. J. Aust. Math. Soc., Ser. A 42 (1987), 5-30.

[4] W. H. Haemers, H. Kharaghani, M. A. Meulenberg, Divisible design graphs, J. Combin. Theory Ser. A 118 (2011), 978-992.

[5] D. G. Higman, Coherent configurations. I. Rend. Sem. Mat. Univ. Padova 44 (1970), $1-25$.

[6] D. G. Higman, Coherent configurations, Part 1: Ordinary representation theory, Geom. Dedicata 4 (1975), 1-32.

[7] H. Kharaghani, New class of weighing matrices, Ars. Combin. 19 (1985), 69-72.

[8] H. Kharaghani, S. Sasani, S. Suda, A strongly regular decomposition of the complete graph and its association scheme, Finite Fields Appl. 48 (2017) 356-370.

[9] H. Kharaghani, R. Torabi, On a decomposition of complete graphs, Graphs and Combin., (2003) 19 519-526.

[10] M. Klin, S. Reichard, A. Woldar, Siamese objects, and their relation to color graphs, association schemes and Steiner design, Bull. Belg. Math. Soc. 12 (2005), 845-857.

[11] M. E. Muzychuk, V-rings of permutation groups with invariant metric, Ph.D. thesis, Kiev State University, 1987.

[12] P.-H. Zieschang, An algebraic approach to association schemes. Lecture Notes in Mathematics, 1628. Springer-Verlag, Berlin, 1996. xii+189 pp. 